\title{
Real-time Monitoring System and Method of PFC Capability of Power Grid Based on Data Analysis
}

\author{
Jun $\mathrm{Li}^{1,{ }^{*}}$,WeiWei Miao ${ }^{2}$, and WenDong Zhang ${ }^{2}$ \\ ${ }^{1}$ State Grid Shandong Electric Power Research Institute, Jinan, China \\ ${ }^{2}$ State Grid Shandong Electric Power Company, Jinan, China
}

\begin{abstract}
The development of wind power and other new energy sources has a great impact on the stability of power system frequency. By analyzing the characteristics of the primary frequency control(PFC) assessment standard of the power grid, one real-time monitoring method of the unit's PFC capability of the power grid based on data analysis is proposed. The Power network dispatching department can fully grasps the overall frequency regulation capability of the operating units. It can improve the grid's response methods to deal with high power gaps and ensure the safe and stable operation of the grid.
\end{abstract}

\section{Introduction}

Since the 21 st century, with the exhaustion of coal, oil etc. fossil energy and the increasing demand for environmental protection, clean and pollution-free renewable energy has attracted close attention of experts and scholars at home and abroad. With the continuous vigorous development of China's wind power industry, the penetration rate of wind power in some provinces continues to increase, and wind power has a serious impact on the safety and stability of the power system due to its volatility and intermittent nature. At present, most domestic wind turbines are variable-speed wind turbines. The speed of such generators is decoupled from the system frequency[1-3]. The wind turbines cannot provide conventional inertial response for the power system, which will cause the frequency of the power system including wind power to decrease under the same disturbance and has a serious impact on power system frequency regulation.

At present, the frequency regulation means of power grid mainly include primary frequency control(PFC), secondary frequency regulation and tertiary frequency regulation. All kinds of regulation means cooperate to ensure the frequency stability of power system on different time scales. As the first line of defense of frequency adjustment, PFC undertakes the important task of rapidly supplementing or absorbing active power to restrain the large frequency changes of the power grid[4,5]. The stable frequency of the power system in China is required to be $50 \pm 0.2 \mathrm{~Hz}$. China mainly relies on thermal power units for frequency modulation compensation. The PFC function is an inherent function of thermal power units. It mainly uses boiler's heat storage. In the event of an abnormality in the grid, it quickly responds to the requirements of the grid to make up for the grid load gap and stabilize the grid frequency. The PFC responds quickly to system frequency changes. According to IEEE statistics, the integrated primary regulation characteristic time constant of the power system is generally about 10 seconds. Since the chemical energy in the combustion system has not changed, as the heat storage decreases, the generator power will return to its original level. At present, the digital electro-hydraulic control system(DEH) of thermal power units has been widely used, so that the PFC function of the generator set is no longer an inherent adjustment feature of the speed control system, but can be artificially changed and switched on. The PFC will cause frequent fluctuations in the speed regulation system and the thermal system, but power plants pay attention to the stable operation of the generator unit and are unwilling to participate in the primary frequency regulation[6-8]. Therefore, the power plant cuts off the PFC function of the unit. The adjustment function results in that the primary frequency modulation capability of the entire power system is always at a very low level, and will cause incorrect actions of the low-frequency load shedding device, and endanger the safe and stable operation of the power system. Therefore, an accurate and real-time evaluation of the primary frequency modulation capability of the generator unit in the power system can effectively increase the quality of the safe and stable operation of the power system.

\section{PFC Performance Assessment}

PFC is related to the safety of the power grid, and it is necessary to evaluate and evaluate its operation.

In the existing frequency supervision system, it is mainly realized by the grid dispatching department's policy to regulate $\mathrm{PFC}$ performance.

There are two types of PFC evaluation methods for thermal units. The first type of method is mainly to calculate the characteristic parameters related to the grid-

* Corresponding author: lijun_sdu@hotmail.com 
connected PFC performance based on the output characteristics of the generator, including the governor's differential coefficient, PFC dead zone and delay time, etc.. The second type of method mainly evaluates the PFC of the unit by calculating the commissioning rate, the correct action rate and combining the power contribution $[9,10]$. It combine the electricity price to calculate the unit penalty coefficient, and use the penalty coefficient to reward and punish the unit with different PFC performance conditions. The evaluation is based on the output curve of the unit and the frequency of the power grid. At present, the second method is commonly used in actual operation.

\subsection{PFC commissioning rate assessment}

Grid-connected generating units must put in the PFC function. When the grid frequency fluctuates, they should automatically participate in the PFC function. Gridconnected power plants must not arbitrarily withdraw from the PFC function of the unit. The monthly commissioning rate of $\mathrm{PFC}$ should reach $100 \%$.

$$
\begin{aligned}
& \lambda=\text { monthly commissioning rate of } P F C \\
& =\frac{\text { monthly commissioning time of } P F C}{\text { monthly grid-connected operation time }} \times 100 \%
\end{aligned}
$$

The assessment electric quantity according to the monthly commissioning rate of PFC is

$$
(1-\lambda) \times P_{N} \times 10 \times \alpha_{P F C}
$$

Where, $P_{N}$ is the unit rated power. $\alpha_{P F C}$ is PFC assessment coefficient, the value is usually 3 .

\subsection{PFC correct action rate assessment}

When one unit is connected to the grid, during an integral period when the grid frequency crosses the dead zone of the unit's PFC, if the unit's PFC function contribution is positive, then the statistics is that the unit has a correct operation for PFC, otherwise, it is an incorrect operation. The calculation formula for the monthly correct action rate is

$$
\begin{aligned}
& \lambda_{\text {action }}=\text { monthly correct action rate } \\
& =f_{\text {correct }} /\left(f_{\text {correct }}+f_{\text {wrong }}\right) \times 100 \%
\end{aligned}
$$

In the formula, $f_{\text {correct }}$ is the number of correct actions per month. $f_{\text {wrong }}$ is the number of incorrect actions per month.

The correct action rate of PFC is evaluated by the unit. For one unit with a monthly correct operation rate of less than $80 \%$, the monthly assessment of power is

$$
\left(80 \%-\lambda_{\text {action }}\right) \times P_{N} \times 2 \times \alpha_{P F C}
$$

Where, $P_{N}$ is the unit rated power. $\alpha_{P F C}$ is PFC assessment coefficient, the value is usually 3 .

\subsection{Electricity contribution index}

As shown in Fig. 1, curve 1 is the frequency value, curve 2 is the ideal action amplitude of PFC, and curve 3 is the practical action amplitude. The actual electricity contribution and the ideal electricity contribution can be obtained from curve 2 and curve 3 . The electricity contribution index should exceed a certain ratio square to meet the grid assessment requirements.

$$
Q_{\text {Actual }}=H=\frac{\int \Delta P(\Delta f, t) \Delta t}{3600}=\frac{1}{3600} \times \sum_{t}\left(P(t)-P_{0}\right)
$$

Where, $Q_{\text {Actual }}$ is actual electricity contribution. $P_{0}$ is the unit's actual output when the frequency variation exceeds the dead zone. $P(t)$ is the unit's actual output during $\mathrm{PFC}$ operation period. $\Delta f$ is the frequency deviation between the actual frequency and PFC dead zone.

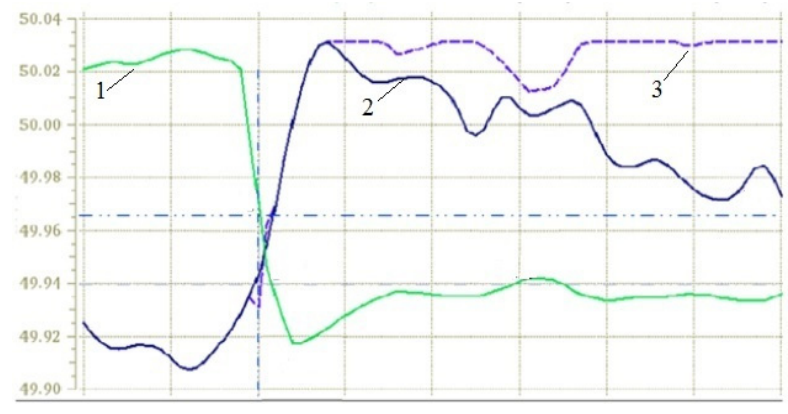

Fig. 1. Schematic diagram of PFC performance assessment for thermal units

$$
Q_{\text {Ideal }}=\frac{\int \Delta P_{N}(\Delta f, t) \Delta t}{3600}=-\frac{1}{3600} \times \frac{P_{N}}{f_{N} \times \delta} \times \sum_{t} \Delta f
$$

Where, $Q_{\text {Ideal }}$ is ideal electricity contribution. $P_{N}$ is the unit rated power. $f_{N}$ is the unit rated frequency $50 \mathrm{~Hz} . \delta$ is the speed governing droop, the value is $4 \%-6 \%$.

$$
Q \%=\frac{Q_{\text {Actual }}}{Q_{\text {Ideal }}} \times 100 \%
$$

The electricity contribution index $Q \%$ refers to the percentage of the unit's actual electricity contribution to the ideal electricity when the frequency change exceeds the limit of the PFC dead zone.

\section{Existing Problems and Optimization}

\subsection{Existing Problems}

At present, because the PFC capability of grid-connected units cannot be effectively predicted and monitored, when large-scale load fluctuations occur in the grid, such as external DC blocking, which causes large power losses, the grid dispatching department can only rely on power gaps to quickly cut off the corresponding line load. This method is likely to cause secondary accidents such as power outages of important loads and large-scale residents power outages, resulting in greater social impact. It is not only detrimental to normal production and operation, but also detrimental to the safety and stability of the power grid. At the same time, with the development of the internet of energy, the number of units participating in frequency modulation is relatively reduced in the developed southeast regions where the proportion of 
external power is gradually increasing, which will place higher requirements on the PFC capabilities of the units on the network.

\subsection{Optimization and Improvement}

In response to the above problems, one real-time monitoring method and system for the PFC capability of the power grid based on data analysis is proposed to evaluate the PFC capability of the unit in real time, and then fully grasp the overall PFC capability of the operating unit.

\subsubsection{Real-time monitoring method}

The real-time monitoring method for the PFC capability of the power grid specifically includes the following steps:

- Step 1, the summary statistics of the PFC capability of the grid-connected frequency modulation unit in each load interval.

- Step 2, analyze and judge the operating status of the grid-connected frequency modulation unit. If the unit is running, analyze the load interval where its current load is located, and give the PFC space value corresponding to the current load interval. If it is not running, set the PFC space value corresponding to all load intervals of the unit to 0 .

-Step 3, the real-time PFC space statistics of the overall grid. Add the PFC space values given by all units in the regional power grid at the current moment to obtain the overall PFC space of the current grid.

In step 1, for the grid-connected frequency modulation units in the regional power grid, according to the installed capacity of the unit, $50 \%-100 \% \mathrm{P}_{\mathrm{N}}$ is divided into 5 load ranges, which are carried out separately according to the national grid's guide of primary frequency control test and performance acceptance for thermal power generating units. In the disturbance test, the upper and lower limits of PFC in a different load interval are respectively calculated.

\subsubsection{Real-time monitoring system}

The real-time monitoring system for the PFC capability of the power grid is shown in the Fig. 2.

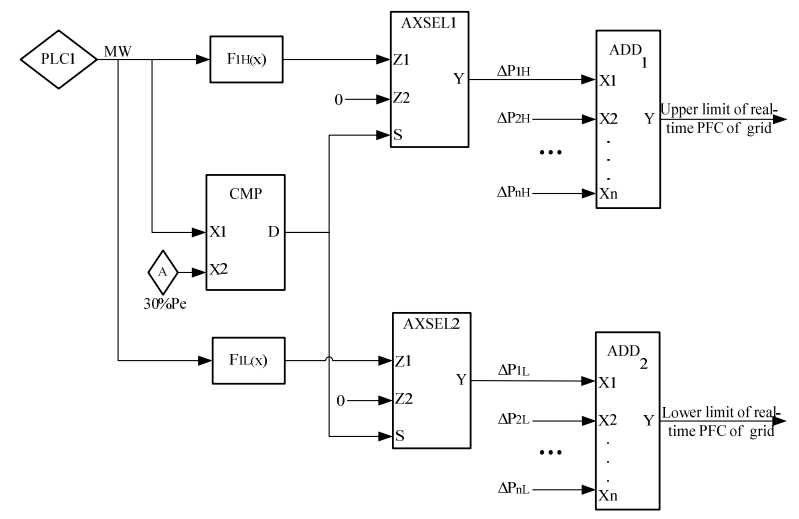

Fig. 2. Real-time monitoring system

The input signals of the comparator module CMP are the unit's real-time load value and the set percentage of the unit's rated power respectively, that is, when the unit's real-time load is considered to be greater than the set percentage of the unit's rated power, it is the unit operating state. The second input terminals of the first selector module AXSEL1 and the second selector module AXSEL2 are both set to zero. The first function setting module $\mathrm{F}_{1 \mathrm{H}}(\mathrm{x})$ is used to generate the upper limit value function of the primary frequency modulation of the unit in different load intervals based on the statistics of the disturbance test, that is, the maximum increase capacity of the primary frequency modulation of the unit. The second function setting module $\mathrm{F}_{1 \mathrm{~L}}(\mathrm{x})$ is used to generate the lower limit value function of the primary frequency modulation of the unit in different load intervals, which is the maximum reduction capacity of the primary frequency modulation of the unit based on the statistics of the disturbance test.

The signal collector module sends the collected realtime load value of the unit PLC1 to the function setting module $\mathrm{F}_{1 \mathrm{H}}(\mathrm{x})$, the comparator module $\mathrm{CMP}$, the function setting module $\mathrm{F}_{1 \mathrm{~L}}(\mathrm{x})$, and the function setting module $\mathrm{F}_{1 \mathrm{H}}(\mathrm{x})$ and $\mathrm{F}_{1 \mathrm{~L}}(\mathrm{x})$, respectively, obtain the upper and lower limits of real-time PFC of the unit at this time.

The real-time load value of the unit is compared with $30 \% \mathrm{P}_{\mathrm{N}}$ in the comparator module CMP. If the unit is in the running state, the real-time load must be greater than $30 \% \mathrm{P}_{\mathrm{N}}$. At this time, the comparator module CMP outputs the switch value Signal 1, this signal is respectively connected to the setting terminal $\mathrm{S}$ of the selector modules AXSEL1 and AXSEL2, and their respective outputs are respectively switched to the $\mathrm{Z} 1$ terminal, that is, the unit's real-time PFC upper limit and lower limit $\triangle \mathrm{P}_{1 \mathrm{H}}$ and lower Limit $\triangle \mathrm{P} 1 \mathrm{~L}$. If the unit is in the stop state, the realtime load must be less than $30 \% \mathrm{P}_{\mathrm{N}}$. At this time, the comparator module CMP outputs the switch signal 0 , which is connected to the selector modules AXSEL1 and AXSEL2's terminal S, switch their respective outputs to terminal Z2, that is, set the upper limit and lower limit of the unit's real-time PFC to constant 0 .

\subsubsection{Application}

According to the China State Grid's criterion Q/GDW 669-Guide of primary frequency compensation test for thermal power generating units, when the $\mathrm{P}_{\mathrm{N}}$ is less than $250 \mathrm{MW}$, the max frequency compensation load is $10 \% \mathrm{P}_{\mathrm{N}}$. When $\mathrm{P}_{\mathrm{N}}$ is more than $250 \mathrm{MW}$ and less than $350 \mathrm{MW}$, the max frequency compensation load is $8 \% \mathrm{P}_{\mathrm{N}}$. When $\mathrm{P} 0$ is more than $350 \mathrm{MW}$ and less than $500 \mathrm{MW}$, the $\max$ frequency compensation load is $7 \% \mathrm{P}_{\mathrm{N}}$. When $\mathrm{P}_{\mathrm{N}}$ is more than $500 \mathrm{MW}$, the max frequency compensation load is $6 \% \mathrm{P}_{\mathrm{N}}$.

Taking one $300 \mathrm{MW}$ thermal power unit in north China as an example, the minimum theoretical upper limit of $\mathrm{PFC}$ is $8 \% * 300 \mathrm{MW}=24 \mathrm{MW}$, and the lower limit is 24MW. However, in actual operation, due to the stable combustion demand of the low-load section and the insufficient main steam pressure in the high-load section, the actual PFC capability cannot reach the theoretical upper and lower limits. 
Table 1. Actual upper and lower limit of frequency modulation

\begin{tabular}{|c|c|c|c|}
\hline Load & Power & $\begin{array}{c}\text { Actual Upper } \\
\text { Limits }\end{array}$ & $\begin{array}{c}\text { Actual Lower } \\
\text { Limits }\end{array}$ \\
\hline $50 \% \mathrm{P}_{\mathrm{N}}$ & 150 & 22 & -21 \\
\hline $60 \% \mathrm{P}_{\mathrm{N}}$ & 180 & 23 & -23 \\
\hline $70 \% \mathrm{P}_{\mathrm{N}}$ & 210 & 24 & -24 \\
\hline $80 \% \mathrm{P}_{\mathrm{N}}$ & 240 & 24 & -24 \\
\hline $90 \% \mathrm{P}_{\mathrm{N}}$ & 270 & 23.5 & -23 \\
\hline $100 \% \mathrm{P}_{\mathrm{N}}$ & 300 & 24 & -24 \\
\hline
\end{tabular}

The actual PFC upper and lower limit value are shown in Fig. 3.

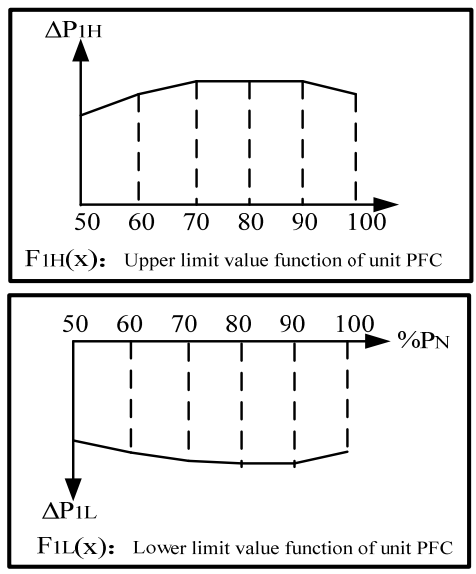

Fig. 3. Limit value function of unit PFC

According to the standard, after the actual lower limit of the PFC of each unit is obtained through the conventional disturbance test, and the cumulative sum is calculated according to its operating status, the real-time PFC space of the power grid can be obtained, as shown in Fig.4. Line 1 is the theoretical upper limit, line 2 is the actual limit, line 3 is the real-time load value, line 4 is the actual lower limit, line 5 is the theoretical lower limit.

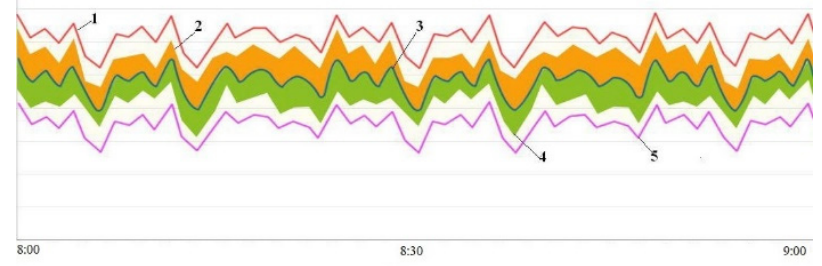

Fig. 4. Real-time PFC space of the power grid

\section{Conclusion}

The PFC in the control area is related to the safety of the power network, but the existing frequency management standards can not conduct good supervision and evaluation of the PFC. Therefore, it is necessary to formulate the corresponding control evaluation standard for the PFC process, so as to standardize the technical specification of all levels of power production departments. Aiming at the problem that the frequency modulation capability of grid-connected operating units cannot be dynamically monitored, the disturbance test realizes the summary analysis of the PFC capacity in each load interval of the unit, which can effectively monitor the PFC status of each grid-connected frequency modulation unit and realize effective supervision management. Through the analysis and judgment of the PFC capability of a single unit through grid dispatching, we can fully grasp the overall frequency regulation capacity of the units in operation, and carry out targeted line removal of the corresponding range, to provide guarantee for the safe and stable operation of the grid, and to ensure the majority of users safe and effective operation of electrical equipment.

\section{Acknowledgements}

The project is supported by the State Grid Shandong Electric Power Company fund 2020A-072 Multi-type power supply network source coordinated safety support and cooperative control technology (520626190022).

\section{References}

1. You R, Barahona B, Chai J, et al. Improvement of grid frequency dynamic characteristic with novel wind turbine based on electromagnetic coupler. Renewable Energy, 113:813-821(2017)

2. Zhang F, Hu Z, Xie X, et al. Assessment of the Effectiveness of Energy Storage Resources in the Frequency Regulation of a Single-Area Power System. IEEE Transactions on Power Systems, 32(5):3373-3380(2017)

3. Zhang Heng, Cheng Haozhong, Zeng Pingliang, etal. Generation and transmission expansion planning considering economy and safety. Automation of Electric Power Systems, 41(21):62-69(2017)

4. YIN Feng. Test and research on CCS-joined primary frequency regulation of thermal power units. Electric Power, 38(3):22-24(2005)

5. Dai Yiping, Zhao Ting, Tian Yunfeng. Research on the primary frequency control characteristics of generators in power system. Second IEEE Conference on Industrial Electronics and Applications, 569574(2007)

6. Zheng Tao, Gao Fuying. On-line monitoring and computing of unit PFR characteristic parameter based on PMU. Automation of Electric Power Systems, 33(11):57-61(2009)

7. Yu Daren, Guo Yufeng. The primary frequency regulation stability of parallel turbo-generators. Proceedings of the CSEE, 20(9):59-63(2000)

8. Li Duanchao, Chen Shi, Chen Zhongyuan. Real-time measurement and reward method of the efficiency of generator unit primary frequency regulation. Automation of Electric Power Systems, 28(2):7072(2004)

9. Zhu Wei, Tan Xiyi, Tang Yingjie. Analysis and 
research on primary frequency modulation of the turbine generation unit. Automation of Electric Power Systems, 32:41-44(2008)

10. Wu Xin, Wu Ning, Sun Haitao, Ren Lin. Practice of improving the performance of primary frequency regulation of thermal power unit. Shandong Electric Power, 45:65-68(2018) 